\title{
Chromatic Orthogonality in Organic Synthesis
}

\author{
Christian G. Bochet*
}

Department of Chemistry, University of Fribourg, 9 Chemin du Musée, CH-1700 Fribourg, Switzerland. Fax: +41-26-300 9738. Email: Christian.Bochet@unifr.ch

\begin{abstract}
:
A major challenge in organic synthesis is the selective reaction of a functional group in the presence of others. This can be achieved by using an appropriate reagent, tuned to react exclusively at the desired centre. An alternate approach would be to use a single reagent, and to transmit from the outside the information as to where it should react. This account describes the use of light as a controlling element; indeed, in addition to the intensity, changing the wavelength gives an additional handle to direct the chemoselectivity.

1 Introduction

2 Background

$3 \quad$ Initial attempts

4 Energy transfer

5 Photocleavable linkers

6 Real orthogonality

7 Photoacylation

$8 \quad$ Outlook
\end{abstract}

Key words: Photochemistry, Protecting groups, Cleavage, Chromophores

\section{$1 \quad$ Introduction}

Ever since the development of the laser, the quest to use light to control the future of matter has been one of the Holy Grails of Chemistry. ${ }^{1}$

Since the dawn of organic synthesis at the end of the 19th century, one of the most actively sought goals in reactions is selectivity, i.e. favouring one over many possible processes.

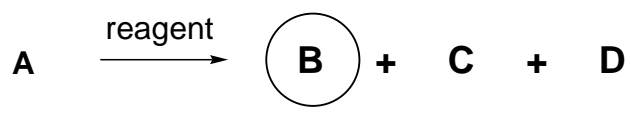

Scheme 1

Let us take the example of the selective addition of a methyl group at to an aldehyde (Scheme 2). In the absence of a directing factor, it is obvious that the addition will occur at both faces of the carbonyl group with equal probability, hence leading to a racemic mixture. One way of altering this ratio is to introduce a chiral auxiliary on a substituent, hence differentiating the two faces (case b). Another approach, probably more flexible, would be to introduce the chiral element on the reagent itself, so that it can choose on which face the carbonyl will be attacked (case c).
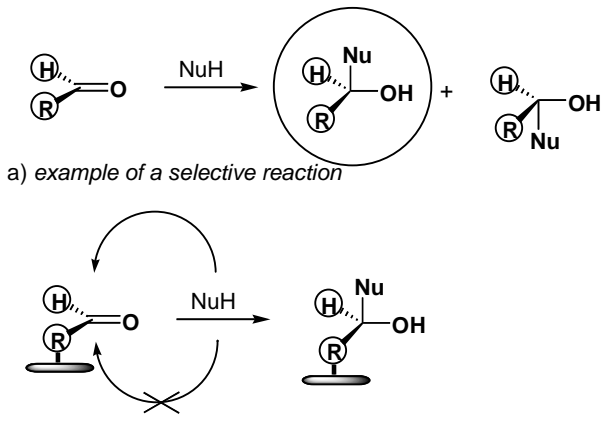

b) information located on the substrate

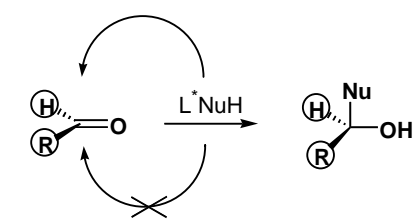

c) information located on the reagent

Scheme 2

In these two cases, information was introduced into the system; this information shapes the potential energy surface in order to bias the reaction towards one specific direction (Scheme 3). The amount of the chiral element, either stoichiometric or catalytic does not change the fundamentals of the process.

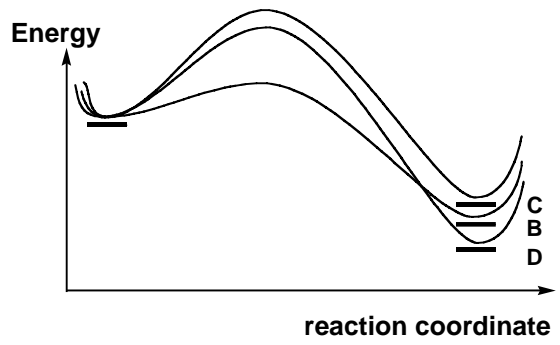

Scheme 3

An even more flexible approach would be to have no information at all within the system, but rather to send it from the outside. In this way, one would tell the system to react one way or another, depending on the external stimulus. This information transmission could in theory be achieved in various ways (magnetic or electric fields, quantum control, microwaves, light); we have decided to use the light. Indeed, light is a very flexible entity, for which wavelength, intensity and polarization can be tuned and therefore could be used to transmit information. In this account, we will describe our initial efforts in this direction by using wavelength tuning. The field is of course in its infancy, and we are still very far from enantioselectivity (many attempts since decades have only 
given very modest results); nevertheless, efficient chemoselectivity has now been reached. Orthogonality is defined as the possibility of making one functional group react selectively in the presence of others under specific conditions, and it is widely used in protecting group chemistry. Hence, a differentiation based on the colour of a light beam could be named chromatic orthogonality. ${ }^{2,3}$

\section{$2 \quad$ Background}

When we initiated this program, very little was known in this area, not even the feasibility of such a concept. Of course, photolabile protecting groups have been known since the 1960's, and it became rapidly apparent that many of them operate at different optimal wavelengths. ${ }^{4}$ Among them, we chose, as initial candidates to test our hypothesis, derivatives of the famous nitroveratrole group 1 (Figure 1).<smiles>COc1cc(CO)c([N+](=O)[O-])cc1OC</smiles>

Figure 1

Introduced in 1970 by Patchornik and Woodward, nitroveratrole esters were shown to release carboxylic acids upon irradiation. ${ }^{5}$ The two methoxy substituents were specifically chosen to enhance the reactivity at $350 \mathrm{~nm}$. This was a promising lead for us, because it suggested that different substituents could shift the optimal wavelength toward lower or higher energy. The only requirement, so far, was the ortho-nitro benzylic moiety, because the mechanism is based on the transfer of a benzylic hydrogen atom to the excited nitro group (Scheme 4):<smiles>[R]C(=O)OC(OC([R])=O)c1cc(OC)c(OC)cc1[N+](=O)[O-]</smiles>

Scheme 4

The exact mechanism of this process is not yet fully understood. In particular, it is not known whether it originates from a singlet or a triplet excited state, and whether the transfer of the benzylic hydrogen should be regarded as radical abstraction or a 1,5-sigmatropic shift. What is firmly established, however, is an ortho-quinoid aci-nitro intermediate and the ortho-nitroso aldehyde side-product. ${ }^{5}$

\section{$3 \quad$ Initial attempts}

We first prepared a series of analogues of $\mathbf{1}$ (2a-g) bearing electron-releasing or -withdrawing groups, either conjugated (i.e. para) with the nitro group or a possible intermediate benzylic radical (Figure 2). ${ }^{6}$<smiles>O=[N+]([O-])c1ccccc1CO</smiles>

2a<smiles>O=[N+]([O-])c1ccc(Cl)cc1CO</smiles>

2c<smiles>O=[N+]([O-])c1ccc([N+](=O)[O-])c([N+](=O)[O-])c1</smiles>

$2 \mathrm{e}$<smiles>O=[N+]([O-])c1ccc(N2CCCC2)cc1CO</smiles>

Figure 2<smiles>O=[N+]([O-])c1cc(Cl)ccc1CO</smiles>

2b<smiles>O=[N+]([O-])c1cc(Br)ccc1CO</smiles>

2d<smiles>O=[N+]([O-])c1cc(-c2ccccc2)ccc1CO</smiles>

In a series of test experiments, we simply photolyzed solutions of the carbamate derivative $\mathbf{2} \mathbf{a}-\mathbf{g}$ in acetonitrile at various wavelengths in a Rayonet apparatus, and measured the apparent rate of conversion. The observed rates of product formation under certain sets of conditions were found to vary substantially with the wavelength. Unfortunately, the different substituents usually accelerated or slowed down the reaction at all wavelengths (254, 300, 350 and $419 \mathrm{~nm}$ ). Although this observation seems obvious, one has to keep in mind that both the absorbance and the quantum yield are affected by substituents, and not necessarily in the same directions. From our collection of compounds, we could however identify a pair of carbamates (3 and 4) showing an opposite trend of reactivity at high- or low-energy photolysis (Scheme 5).

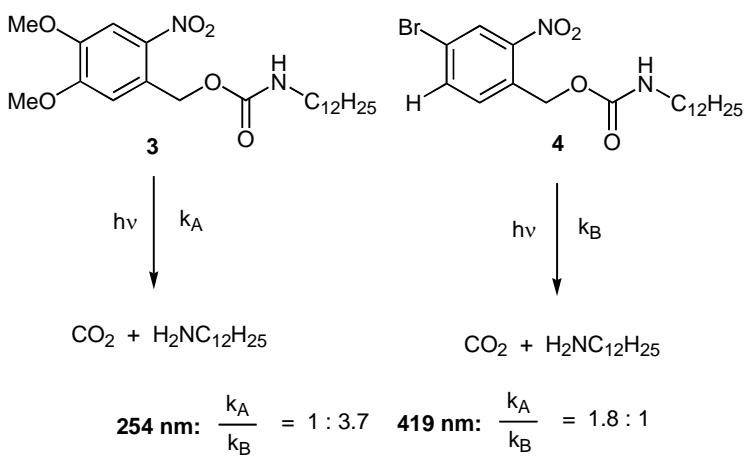

\section{Scheme 5}

Although the rate constant ratio (almost 1:4 at $254 \mathrm{~nm}$ and 2:1 at $419 \mathrm{~nm}$ ) clearly showed a reversal of reactivity at the two wavelengths, the selectivities were obviously too low for a useful synthetic application.

\section{$4 \quad$ Energy transfer}

At this point we realised that by changing substituents on the aromatic ring we would probably never achieve the kind of selectivity we were looking for $\left(\mathrm{k}_{\mathrm{A}} / \mathrm{k}_{\mathrm{B}}>10: 1\right.$ and $<1: 10)$. Hence, we turned our attention to other photolabile groups operating by different mechanisms, more 
prone to require a very different excitation energy. The 3,5-dimethoxybenzyl esters were appealing to us as the absence of functional groups absorbing strongly at lowwavelength suggested a relative inertness at $420 \mathrm{~nm}$. The mechanism, still controversial, was initially proposed to occur by a heterolysis (and later revised as a homolysis followed by a very fast electron transfer) of the benzylic $\mathrm{C}-\mathrm{O}$ bond, leading to a benzylic cation (stabilized in the excited state by the meta-methoxy groups) and a carboxylate anion. ${ }^{7}$ Thus, we performed our standard experiment with such a derivative (Scheme 6):<smiles>CCCCCCCCCCCCCCCCCCC(=O)OCc1cc(OC)cc(OC)c1</smiles>

$$
254 \mathrm{~nm}: \frac{\mathrm{k}_{\mathrm{A}}}{\mathrm{k}_{\mathrm{C}}}=1: 16 \quad 350 \mathrm{~nm}: \frac{\mathrm{k}_{\mathrm{A}}}{\mathrm{k}_{\mathrm{C}}}>100: 1
$$

\section{Scheme 6}

The excellent ratio of reaction rates between $\mathbf{3}$ and $\mathbf{5}$, which were now clearly inverted at 254 and at $350 \mathrm{~nm}$, gave us new hope that our concept could work. However, in order to really prove it, we needed to test a 1:1 mixture of both reactants. It was a major disappointment to see that the good selectivity we had just observed was reduced to none! Both derivatives indeed are photolyzed at very different rates at $254 \mathrm{~nm}$ only when they are in individual vials (Scheme 7, dashed lines); the difference nearly vanishes when $\mathbf{3}$ and $\mathbf{5}$ are irradiated together (full lines). Most probably, energy or electron transfer from the dimethoxybenzyl alcohol derivative to the nitrobenzylalcohol derivative (absorbing at lower energy and potentially an electron acceptor) completely scrambles the initial site of absorption.

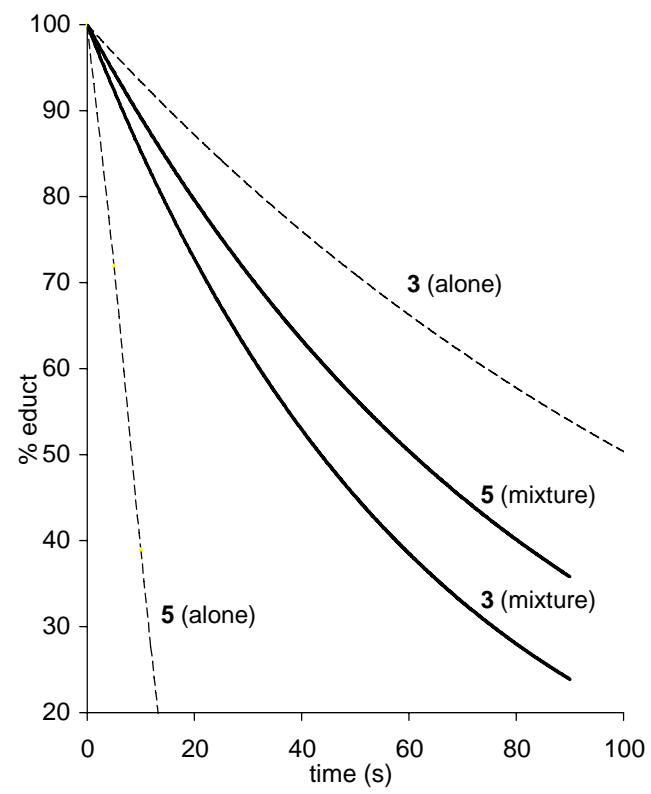

Scheme 7: Photolysis rates at $254 \mathrm{~nm}$

5

\section{Photocleavable linkers}

One obvious way of circumventing the problem was to first remove the low-energy labile group, and then photolyze the remaining group at higher energy. A discussion with Bernd Giese (University of Basel) revealed that he had just developed the new photolabile pivaloylpropanediol group 7 with virtually no absorbance at 350 nm. ${ }^{8}$ Excited by this possibility, we immediately initiated a collaboration, which resulted in a test compound, the simple aliphatic diester $\mathbf{8}$, with a nitroveratryl derivative at one terminus, and the pivaloyl derivative developed in Basel (Scheme 8).<smiles>COc1cc(COC(=O)CCCC(=O)O)c([N+](=O)[O-])cc1OC</smiles><smiles>COc1cc(COC(=O)CCCC(=O)OCC(C)(O)C(=O)C(C)(C)C)c([N+](=O)[O-])cc1OC</smiles>

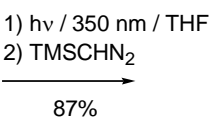<smiles>COC(=O)CCCC(=O)OCC(C)(O)C(=O)C(C)(C)C</smiles>

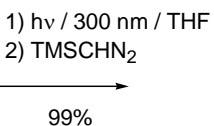<smiles>COC(=O)CCCC(=O)OC</smiles>

Scheme 8

Indeed, photolysis of this diester at $360 \mathrm{~nm}$ smoothly gave the monoester 9 resulting from the cleavage of the nitroveratryl group, in a $87 \%$ yield, with only $2 \%$ of the wrong regiochemistry. ${ }^{9}$ Subsequent photolysis at $300 \mathrm{~nm}$ gave the dimethylester $\mathbf{1 0}$ resulting from the cleavage of the remaining group. Of course, the price to pay for this is a significantly limited flexibility. Successive deprotection with the same reagents involving harsher and harsher conditions is called modulated lability. ${ }^{10}$ Despite obvious limitations, modulated lability is still highly useful, for example in solid-phase organic synthesis (SPOS). Indeed, a photolabile linker that is very robust against chemical reagents, and which can be removed only by high-energy light, this would allow for mild photochemical reactions on the chain. This is what we developed, still in collaboration with Bernd Giese, in a two-wavelength solid phase peptide synthesis. Both the terminus liberation (prior the coupling of the next amino acid) and the linker cleavage were effected by irradiation, but at two different wavelengths. As a classical example, Leu-Enkephalin was prepared by this procedure, in an overall yield of $55 \%$ (Scheme 9). ${ }^{9}$ 

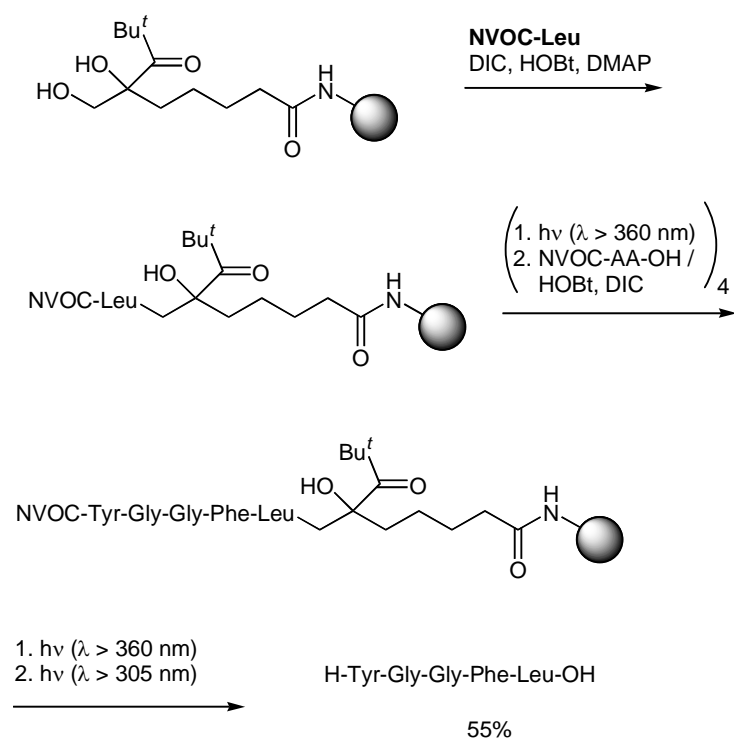

Scheme 9

\section{Real orthogonality}

Despite this encouraging success, we were still aiming at developing real chromatic orthogonality. It was therefore of critical importance to establish whether quenching problem was specific to the pair of protecting groups we had selected, or rather a more general phenomenon for all bichromophoric systems. Thus we examined closely the reaction kinetics compounds $\mathbf{3}$ and $\mathbf{4}$, both for individual solutions (dotted lines, Scheme 10) and for 1:1 mixtures (full lines).

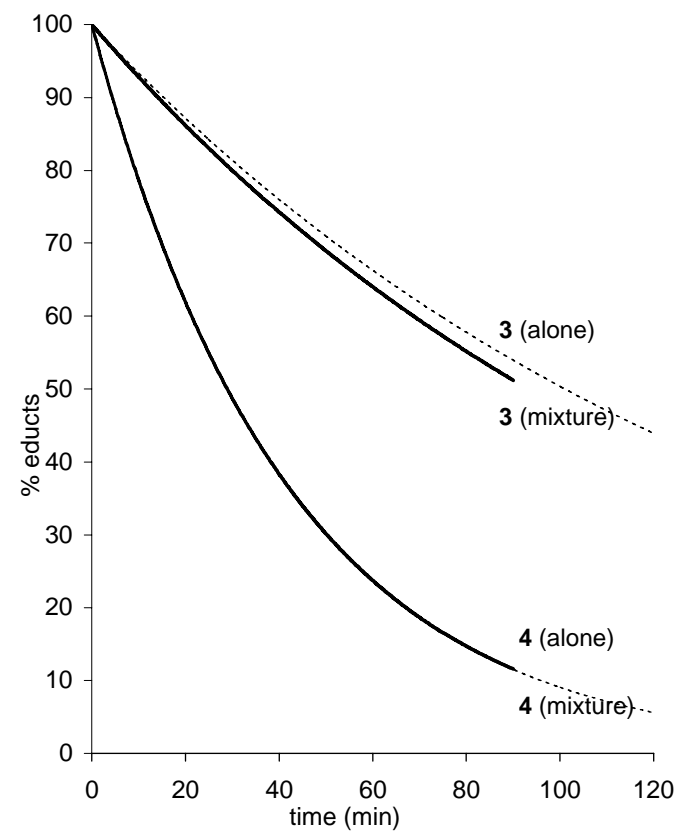

Scheme 10. Photolysis rates at $254 \mathrm{~nm}$

We were pleased to see that, in this case, the reaction rates were exactly the same (within experimental error) for individual solutions and mixtures. ${ }^{6}$ The conclusion is that there was no energy transfer, which gave us hope that it would indeed be possible to make one subset or the other of a mixture react independently by selecting the appropriate wavelength. This was verified by monitoring the mixture composition over time at the two different wavelengths. Scheme 11 shows the ${ }^{1} \mathrm{H}$-NMR spectra of the benzylic protons of the $\mathbf{3} / \mathbf{4}$ carbamate mixture after increasing irradiation times at both wavelengths. It shows unambiguously that indeed, although to a modest extent, only one of the substrates does react. Hence, for the first time, it was possible to select individual molecules from a group by an external stimulus. Far from any useful application, it was nevertheless the proof of principle we were looking for.
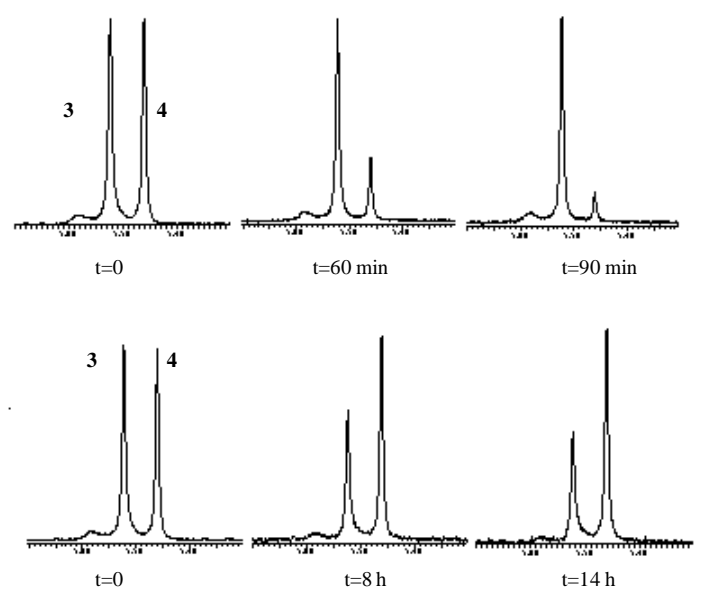

Scheme 11

Once the conceptual validity was established, all what we had to do was to find a pair of groups with highly differential photolysis rates, with no or minimal energy transfer! A footnote in a 1964 paper by Sheehan gave us a lead. $^{11 \mathrm{a}}$ 3',5'-Dimethoxybenzoin esters underwent very efficient photocleavage to give benzofurans and carboxylic acids. This reaction was not affected by added piperilene or naphthalene; a clear sign of a very short-lived excited state and hence minimal tendency for energy transfer. While we had no interest in making benzofurans, the released carboxylic acid made this benzoin a potentially useful protecting group. This potential was actually exploited by the same authors a few years later. ${ }^{11 \mathrm{~b}}$ In addition to its absence of quenching, the benzoin derivative showed a high quantum yield for photocleavage (0.74) and strong absorbance below $300 \mathrm{~nm}^{11 \mathrm{c}}$ Hence, we checked it against the nitrobenzyl alcohol derivative, this time immediately as a 1:1 mixture of esters $\mathbf{1 1}$ and $\mathbf{1 2}$ bearing a slightly different side chain (Scheme 12). While this had no effect on the photolysis rate, it would allow us to trace the origin of the released acid (or methyl ester after methylation). 


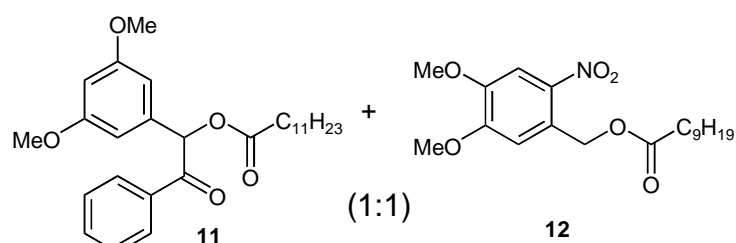

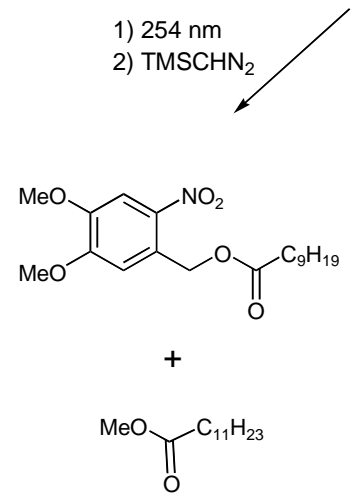

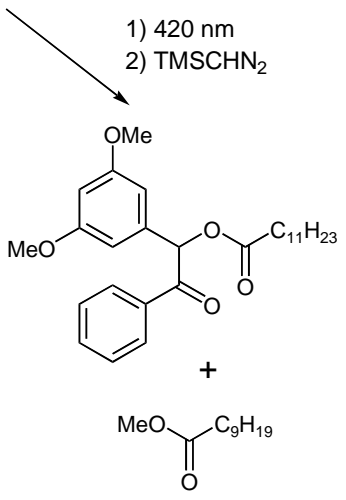

$\mathrm{C}_{11} / \mathrm{C}_{9}: 90: 10(\mathrm{GC})$

Scheme 12

Subjecting a 1:1 mixture of the 3',5'-dimethoxybenzoin ester 11 and the $o$-nitroveratryl ester 12 to $254 \mathrm{~nm}$ irradiation resulted in the preferential deprotection of the benzoin (up to $90 \%$, quantified by GC analysis of the methyl ester, as a C9/C11 ester ratio), whereas the same mixture irradiated at $420 \mathrm{~nm}$ gave up to $85 \%$ of the other ester. ${ }^{2}$ This time, we could really, and in a synthetically relevant manner, select one partner of a mixture by our choice of the wavelength of irradiation. In other words, the groups were orthogonal, not in the classical chemical way, but in a chromatic way (based on the colour of the incident light). If the problem of energy transfer could be dealt with, what would happen when both groups were on the same molecule? Closer proximity would favour energy transfer (which is very distance dependent, according to both the Dexter or Förster mechanisms).<smiles>COc1cc(OC)cc(C(OC(=O)CCC(=O)OCc2cc(OC)c(OC)cc2[N+](=O)[O-])C(=O)c2ccccc2)c1</smiles><smiles>COC(=O)CCC(=O)OCc1cc(OC)c(OC)cc1[N+](=O)[Te]C(=O)OC</smiles>

Scheme 13

We were pleased to see that on a 1,7-diester, no significant erosion of selectivity could be observed, i.e. the chromatic orthogonality was preserved in the intramolecular case. We then gradually reduced the length of the tether, in order to see whether there would a critical distance below which there would be energy transfer. Surprisingly this was not the case, and even the oxalate could be differentiated without significant problem (apart from the inherent chemical instability of the monoester). ${ }^{3}$

\begin{tabular}{ccc}
$\mathrm{n}$ & $254 \mathrm{~nm}: \mathbf{1 4}^{\mathrm{a}}$ & $420 \mathrm{~nm}: \mathbf{\% 1 5}^{\mathrm{a}}$ \\
\hline 5 & $92 \%(70 \%)$ & $70 \%(70 \%)$ \\
4 & $78 \%(69 \%)$ & $95 \%(68 \%)$ \\
3 & $96 \%(70 \%)$ & $85 \%(81 \%)$ \\
2 & $94 \%(81 \%)$ & $85 \%(72 \%)$ \\
1 & $94 \%(85 \%)$ & $83 \%(70 \%)$ \\
0 & $86 \%(27 \%)$ & $67 \%(--)$ \\
\hline
\end{tabular}

Table 1. Photolysis of various esters 13. ${ }^{\text {a }}$ Yields estimated by ${ }^{1} \mathrm{H}-\mathrm{NMR}$. In parentheses: isolated yields.

\section{$7 \quad$ Photoacylation}

So far, chromatic orthogonality was shown in the context of photocleavage, i.e. the breaking of bonds (a rather destructive goal). Could this concept be used for making bonds?

The photochemical acylation of nucleophiles is known since the seminal work of Patchornik and co-workers in $1976 .^{12}$ The principle is quite simple: an acyl group substituted by a poor leaving group is normally inert towards nucleophilic attack (e.g. like an amide). On the other hand, a good leaving group makes the acyl centre very electrophilic (like in an acid chloride). Photochemical activation of a leaving group hence triggers the acylation (Scheme 14). This would be a good opportunity to exemplify further the chromatic orthogonality, for instance by transferring one group or another based on the wavelength of the incident light.

$$
\text { no reaction }
$$

Scheme 14

We first optimized the photoacylation of amines with an analogue of Patchornik's first indoline, bearing two nitro groups. Thus, we could prepare amides ${ }^{13}$ and carbamates $^{14}$ in a neutral medium, by simply exposing a stoichiometric mixture of the acylating agent and the amine to UV-light (Scheme 14). More interestingly, by altering the substituent in the C-7 position of the indoline, we could also influence the photochemical behaviour. 
Thus, a $\mathrm{CH}_{2} \mathrm{COOMe}$ group favoured the reaction at 300 $\mathrm{nm}$, whereas a bromo substituent allowed the reaction to proceed at $420 \mathrm{~nm}$. The competitive experiment with a propionyl and an acetyl derivative exemplified this reactivity (Scheme 15). ${ }^{15}$
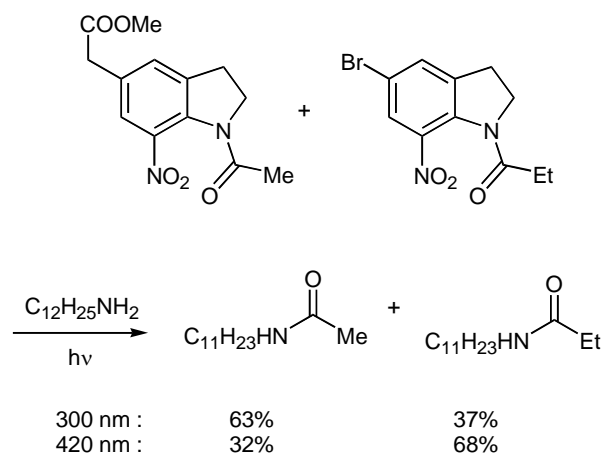

Scheme 15

Although the level of selectivity is still very modest, it is an unambiguous proof that chromatic orthogonality has a much larger field of application than just photolysis of protecting groups. A combined approach, with the introduction of protecting groups and their removal only by photochemical activation is currently being investigated in our laboratories.

\section{Outlook}

We have shown that chromatic orthogonality is indeed a valuable principle for organic synthesis. More importantly, our goal was to demonstrate that photochemical reactions could be as selective as conventional 'dark' reactions, and that subtle levels of control could be attained by carefully choosing the conditions. We are now exploring this fascinating new field, and developing additional types of reactions capable of chromatic orthogonality. ${ }^{16}$ This expansion needs to be done in both ways: new reactions and additional dimensions of the orthogonal space, i.e. more specific wavelengths that could be used. Twophoton absorption and the use of tuneable laser sources are two obvious directions to head for; one should however not expect too much from technology. The current limiting factor is our creativity.

\section{Acknowledgements}

This work could not have been carried out without a group of very talented and dedicated co-workers. I thank warmly my group at the University of Geneva: Céline Helgen, Aurélien Blanc, David Gérard, Dui-Hien Tran, David D'Addonna, Daniel Di Emidio. The collaboration with Basel has also been extremely pleasant and fruitful, and I thank Prof. Bernd Giese and his students Martin Kessler and Ralf Glatthar for their friendly interaction and stimulating work.

\section{References and Notes}

(1) Kohler, B.; Krause, J. L.; Raksi, F.; Wilson, K. R.;
Yakovlev, V. V.; Whitnell, R. M.; Yan, Y. Acc. Chem. Res. 1995, 28, 133-140. This very inspiring article is entitled Controlling the Future of Matter. See also: Zare, R. N. Science 1998, 279, 1875-1879.

(2) Bochet, C. G. Angew. Chem. Int. Ed. 2001, 40, 20712073.

(3) Blanc, A.; Bochet, C. G.; J. Org. Chem. 2002, 67, 5567-5577.

(4) Bochet, C. G. J. Chem. Soc. Perkin I 2002, 125-142.

(5) Patchornik A.; Amit, B.; Woodward, R. B. J. Am. Chem. Soc. 1970, 92, 6333-6335. For a recent mechanistic study, see: Il'Ichev, Y. V.; Schwörer, M. A.; Wirz, J. J. Am. Chem. Soc. 2004, 126, 4581-4595.

(6) Bochet C. G. Tetrahedron Lett.. 2000, 41, 6341-6346.

(7) Chamberlin, J. W.; J. Org. Chem. 1966, 31, 16581660. Pincock, Acc. Chem. Res. 1997, 30, 43-49; Zimmerman, H. E. J. Am. Chem. Soc. 1995, 117, 8988 and references cited.

(8) Peukert, S.; Giese, B. J. Org. Chem. 1998, 63, 90459051. Glatthar, R.; Giese, B. Org. Lett. 2000, 2, 2315-2317.

(9) Kessler, M.; Glatthar, R.; Giese, B.; Bochet, C. G. Org. Lett. 2003, 5, 1179-1181.

(10) Schelhaas, M.; Waldmann, H. Angew. Chem. Int. Ed. 1996, 35, 2056-2083.

(11) a) Sheehan, J. C.; Wilson, R. M. J. Am. Chem. Soc. 1964, 30, 5277-5281. b) Sheehan, J. C.; Wilson, R. M.; Oxford, A. W. J. Am. Chem. Soc. 1971, 93, 7222-7228. c) For more recent work, see: Rajesh, C. S.; Givens, R. S.; Wirz, J. J. Am. CHem. Soc. 2000, 122, 611-618 and references cited.

(12) Amit, B.; Ben-Efraim, D. A.; Patchornik, A. J. Am. Chem. Soc. 1976, 98, 843.

(13) Helgen, C.; Bochet, C. G. Synlett 2001, 1968-1970. See also Nicolaou, K. C.; Safina, B. S.; Winssinger, N. Synlett 2001, 900.

(14) Helgen, C.; Bochet, C. G. J. Org. Chem. 2003, 68, 2483-2486.

(15) Helgen, C.; Bochet, C. G. Manuscript in preparation.

(16) Blanc, A.; Bochet, C. G. J. Org. Chem. 2003, 68, 1138-1141. 